\title{
Experiences in Automatic Assessment on Mass Courses and Issues for Designing Virtual Courses
}

\author{
Lauri Malmi \\ Department of Computer \\ Science and Engineering \\ Helsinki University of \\ Technology \\ Finland \\ Ima@cs.hut.fi
}

\author{
Ari Korhonen \\ Department of Computer \\ Science and Engineering \\ Helsinki University of \\ Technology \\ Finland \\ archie@cs.hut.fi
}

\author{
Riku Saikkonen \\ Department of Computer \\ Science and Engineering \\ Helsinki University of \\ Technology \\ Finland \\ rjs@cs.hut.fi
}

\begin{abstract}
In this paper, we present some experiences on using automatic assessment in large scale courses of introductory programming, data structures, and algorithms over a period of 10 years. Automatic assessment provides an effective method for giving immediate $24 / 7$ feedback service for students of mass courses. A very important factor in the promoting of learning is the possibility to resubmit answers after receiving the feedback. However, our experience shows that the resubmission option is not the only key motivation factor. More important factors include the challenge of exercises and the grading scale or the course assignments. A successful combination of all of these can produce good learning results.
\end{abstract}

\section{Categories and Subject Descriptors}

K.3.1 [Computer Uses in Education]: Computer-assisted instruction (CAI), Distance learning; K.3.2 [Computer and Information Science Education]: Computer science education-programming, data structures and algorithms

\section{General Terms}

Human Factors

\section{Keywords}

automatic assessment

\section{INTRODUCTION}

Constructivism [11] is a learning paradigm that strongly influences current trends in education. Its basic idea - that students build and process their own mental models - has

Permission to make digital or hard copies of all or part of this work for personal or classroom use is granted without fee provided that copies are not made or distributed for profit or commercial advantage and that copies bear this notice and the full citation on the first page. To copy otherwise, to republish, to post on servers or to redistribute to lists, requires prior specific permission and/or a fee.

ITiCSE'02, June 24-26, 2002, Aarhus, Denmark.

Copyright 2002 ACM 1-58113-499-1/02/0006 ...\$5.00. several implications for how courses should be taught. Moreover, the role of the teacher can change radically. It is not enough to give lectures, deal out assignments and exercises, and check examinations. Instead of being a lecturer and a supervisor, the teacher can act as a coach or an expert consultant, which facilitates students' learning process by creating learning environments, giving guidelines, commenting, giving feedback on students work, etc.

This new vision of the teachers' role may work well when the classes are relatively small and the teacher can follow student's individual work closely. Unfortunately, this is not the case in mass courses involving several hundreds of students, which are common in introductory computer science courses at university level. The teacher cannot concentrate on individuals; instead his/her main role focuses on managing and organising courses in addition to lecturing. The situation is no better in virtual courses, which are sometimes seen as "the trend of the future", and the solution to resource problems. In reality, the interaction between the teacher and the students is even slower if the principal mode of communication is writing. Thus, we cannot manage large virtual courses with small human resources, particularly with a round the clock help service.

Should we then give up and hold on to traditional teaching methods? We argue that this is not necessary. At Helsinki University of Technology (HUT), we have been using automatic assessment (AA) as a supporting method for teaching large introductory courses (typically, 300 to 800 students per course). Our experience is that AA is effective for courses involving simple programming assignments and algorithm simulation exercises. By algorithm simulation we mean exercises whereby a student shows how a given algorithm changes a given input data set step by step, and reports the steps and/or the final state of the data structure.

We have been using the TRAKLA system [4, 7] on the Data Structures and Algorithms course since 1991, and the Ceilidh system [2] on several programming courses since 1994. We started with $\mathrm{C}$ and Fortran languages, and adopted Java in 1998. Since 1999 we have been using the SchemeRobo [13] system on the Scheme-based introductory course for computer science majors. Common to the use of all of these systems is that a vast majority of the students can self study the topic with the aid provided by the tools. Per- 
sonal advice (office hours, email service, newsgroups etc.) is provided for the minority who face difficulties with the assignments.

In this paper, we present some experiences and observations that we have made over the past 10 years. We briefly consider where automatic assessment is feasible and review a number of AA systems in Section 2. In Section 3, we present arguments why we regard AA tools as a successful aid for the teacher. In Sections 4 and 5, we present and discuss a number of observations that we have made when gathering data on students' performance. It is inevitable, for example, that students get better results when they are allowed to resubmit their work. However, this is not enough; it is more important to organise the course so that the skills and the challenges of the students coincide. In the conclusion, we summarise the observations, and discuss briefly their significance for the teacher. We additionally predict how AA can be used to monitor students' progress and learning process more closely.

\section{OVERVIEW OF AUTOMATIC ASSESS- MENT}

Automatic assessment is a method in which a computer program aids the teacher in grading students' work, and facilitates the feedback process. It can be semi-automated where the teacher does the assessing but the tool simplifies the process, for example by providing him/her with ready-made forms and by checking the consistency of the grades [5]. In this paper, however, we consider only systems in which the entire assessment process is carried out by the computer program.

Technically, a simple form of AA is the automatic checking of multiple choice questions. However, several more advanced systems exist. Ceilidh [2] is a system which processes student's submitted computer programs. Other similar systems include ASSYST [6] and TRY [12]. Ceilidh compiles the program, executes it using predefined test data sets, and records the output. Since the outputs of different programs may have different textual formats, the teacher defines a set of regular expressions that are used in searching appropriate strings from the output. Based on the search results, Ceilidh informs the student whether has failed or passed a certain test. Moreover, the teacher can control the level of detail sent back to the student.

String matching can additionally be used for non-programming courses. English and Siviter presented a system [3] for a course of introductory computer tools, for checking whether students send their requested email and news messages, understand certain basic concepts, and can construct web pages with certain specific features of HTML.

Clearly, the comparison of program output strings can be erroneous. Therefore, systems comparing program structures directly have been developed. Among these is the Scheme-Robo [13] system, which assesses submitted Scheme functions. Because Scheme is a functional language, it is easy to analyse the Scheme-expression returned by the submitted function directly. Thus, there are no format problems in the comparison. A different approach was used by Baker et al. [1], who presented a tester system for data structures implemented in Java. Their system interacts directly with the data structures, handles responses, and compares the behaviour of students' work with a "reference" implementation.
Quite different methods are needed for checking algorithm simulation exercises $[4,7,9]$, in which the student shows how a given algorithm changes the state of a given data structure step by step. For example, the student could be asked to insert a set of given key values, one by one, into an initially empty red-black tree and report the tree after six insertions and after all the keys have been inserted. The TRAKLA system $[4,7]$ checks the resulting states by comparing the submitted answer to the corresponding model data structure, which is generated by running the algorithm implemented by the teacher. The answers, submitted either by email or by using a graphical user interface, are converted to strings, and several heuristics are used to match them with the model solution. The recently published Matrix system $[9,10]$ compares each state of the submitted data structure to the model solution, thus allowing more detailed information about possible errors. Matrix additionally provides model solutions for the student in the form of stepwise algorithm animations.

\section{AUTOMATIC ASSESSMENT SUPPORT- ING LEARNING}

The principal benefit of AA, based on our experience, is that it enables one to set up enough compulsory exercises for the students and to give them feedback on their work on a large scale. Every year, approximately 1500 new undergraduate students are enrolled in HUT, more than $2 / 3$ of whom take introductory programming courses. Programming in the small is a practical skill. It is important that the students write and test enough programs in order to practise their skills and, in addition, learn such important concepts as data structures and algorithms. Our experience shows that voluntary exercises do not motivate students enough to reach the required skill level. Compulsory assignments do not provide a great deal more unless we can monitor students' progress regularly and, which is more important, give them feedback on their performance. This would be possible in weekly classroom exercises, if we only had enough classroom time and teachers, which unfortunately is not the case. By means of AA, we can partly solve the feedback problem. We do not argue, however, that computerised feedback would generally match expert human feedback. In some cases it does, as we have found in a research work carried out in spring 2001 on our data structures and algorithms course [8]. In general, however, computerised feedback is far better than merely dealing out model solutions with no feedback on students' work.

The second benefit of $\mathrm{AA}$ is that the computer can give feedback at any time and place. Thus, it applies particularly well to virtual courses - it has in effect turned most of our courses partially virtual. We do have lectures and some classroom sessions, but the students do most of their selfstudy on the Web.

The third benefit is that we can allow the students to revise their submissions based on the feedback. By setting up the assignments so that there is no option allowing the use of the trial-and-error method, the student is pushed to rethink his/her solution, and to make hypotheses about what is wrong. This is in effect what constructivism suggests we should do. Moreover, it is notable that in classroom sessions, such a situation is rarely achieved for every student, since the teacher cannot follow and guide the work of all the students at the same time. 
The fourth benefit is that we can personalise assignments, i.e., all students have different assignments, which discourages plagiarism and encourages natural co-operation in solving problems. With human teaching resources, it is practically impossible to achieve this in large courses.

Finally, AA tools save time and money. We feel, however, that they represent an extra resource that helps us to teach better. Moreover, they allow us to direct human resources to work which cannot be automated, such as personal guidance and giving feedback for the more challenging exercises like design assignments.

\section{CASE: DATA STRUCTURES AND ALGO- RITHMS COURSE}

We have been using the TRAKLA system since 1991 on the Data Structures and Algorithms course. The system has since evolved and certain changes in the course requirements have taken place. Throughout this time, the algorithm simulation exercises have been a compulsory part of the course. In addition, most of the assignments have remained unchanged since 1993. The lecturer and teacher-incharge changed only once during this time, in autumn 1996.

In Figure 1, we can see the distribution of grades during the period 1993-2001. There are four categories of students. First, those who achieved less than $50 \%$ of maximum points and failed the exercise part. Second, the students who received $50-90 \%$ of maximum points and passed. Third, those who received more than $90 \%$ of the maximum points and received the maximum grade, and finally those students who solved all the exercises correctly. The number of students increased from 334 in 1993 to 718 in 2000.

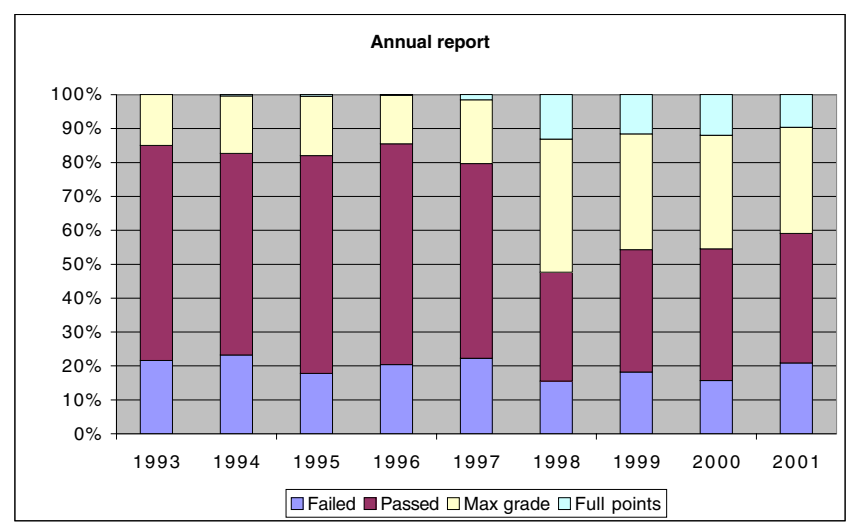

Figure 1: Distribution of grades.

It should be noted, however, that the presented results are not the final results. We required that all student receive a certain minimum number of points for each of the 5 to 6 exercise rounds. Each round typically included exercises related to a specific important course topic, such as sorting, search trees, or graphs. If a student failed to get the minimum from one or more rounds, he/she had the option to pass them by completing extra assignments later. They could also raise their grades. Normally, 50-100 students used this option. However, organisation of the extra assignments has changed quite considerably over the years. Thus, we present the results without these additional points.

\subsection{New Tools and Features}

There are significant changes in the distribution. Up to 1997, the overall distribution remained roughly consistent but thereafter the students achieved far higher grades, particularly in 1998. Subsequently, the results weakened slightly. It is interesting to compare these results and the changes that have taken place. Between 1993 and 1996, both the TRAKLA system and the course requirements remained unchanged. In 1997, however, we introduced two major enhancements to the system. First, we allowed resubmitting of answers. Up to 1996, students had only one chance to submit their solution, and they received the feedback after the deadline for the submission. Second, we introduced a graphical web-based front-end, called TRAKLA-EDIT, for solving assignments. Previously, the only method of submission was to send an email message in a predefined format to the TRAKLA server. All data structures had to be presented as ASCII text, which was somewhat clumsy, for example in the case of trees. TRAKLA-EDIT is a tool that presents the assignments as graphical applets, and allows students to construct the algorithm simulation using simple drag-and-drop operations. They can additionally review their solution sequence by stepping it forth and back before submitting it. The front-end takes care of transforming the required states into the format required by the TRAKLA server. Thus, simple formatting errors can be avoided in the solutions, and visual representations for data structures can be provided.

As can be anticipated, the results for 1997 were somewhat better than for 1996. It is interesting to speculate, however, as to what happened in the following year. On average, students submitted an answer to an assignment 1.45 times in 1997, and 1.47 times in 1998. The proportion of students who used TRAKLA-EDIT was $23.7 \%$ and $42.6 \%$, respectively. These figures do not, however, explain all the changes. A closer examination revealed the following. First, the results of the first 1997 and 1998 submissions compared with 1996 (when only one submission was allowed), were clearly worse. It thus seems that, after the resubmission was allowed, students submitted their first answer more carelessly than before. However, the overall performance was slightly better in 1997 and considerably better in 1998, as can be seen from Figure 1.

When we compared two separate groups of students, those who used only email for submissions, and those who used TRAKLA-EDIT, we observed no differences. Thus, the submission media does not appear to explain the entire change, either.

\subsection{Changes in Course Requirements}

Because we cannot explain the difference between the 1997 results and those of 1998 in this way, the changes in the course contents and organisation in 1998 probably have had a major effect on the results.

First, we dismissed such simple exercises as manipulating stacks and queues, because they were too trivial in graphical form. Instead, we added a number of more challenging exercises, such as inserting items into an AVL tree, a red-black tree, a $B^{+}$-tree, using extendible hashing, and generating minimum spanning trees and shortest path trees.

Second, we introduced another type of exercise, which was submitted on paper and checked manually. These considerably more challenging analytical and constructive exer- 
cises included algorithm analysis and small algorithm design problems.

Third, the grading policy of the simulation exercises was changed. In 1997, if the student's result in the exercises was between 50 and $80 \%$ of maximum, the final grade of the course was the grade of the exam (failed $=0, \ldots, \max =5$ ). If the result was at least $80 \%$, the final grade was the exam grade plus one unit, unless the student failed or achieved $5 / 5$ of the exam. Since 1998 , the home exercise grading was changed: the result 50-60\% corresponded to grade 1, 60-70\% grade 2 etc. Thus, at least $90 \%$ of maximum was required for the best grade, 5 . The proportion of the simulation exercises of the total grade was $30 \%$, that of analytical exercises $30 \%$, and that of the exam $40 \%$.

\subsection{Discussion}

As an overall effect, the course requirements and the course work load were increased quite considerably in 1998. It is interesting that the results were far better under these circumstances. Why did this happen? We assume that two factors played a major role. First, the change in the grading policy, combined with the option to resubmit the exercises, motivated the students to aim at better grades because the exercises had more effect on the final grade. Second, the more advanced exercises raised their motivation, and the learning curve as a whole, i.e., previously, the course had been too easy to pass and did not motivate students to learn more.

Moreover, because of the increase in the work load for both the teachers and the students, we replaced the analytical exercises with a more general half-semester small group project assignment in years 1999 - 2001. During this time, the course results seemed slightly to deteriorate. Thus, it seems that the difficulty of the exercises motivates the students to better performance. We observed this same effect in the research carried out in spring 2001 [8]. Some students on the course did more challenging exercises than the algorithm simulation exercises. Their results in the exam were significantly better than those who did only simulation exercises. On the other hand, their drop-out was high. In 1998 such drop-out did not occur, and we suspect that this is why the students had no comparison group, which performed "easier" tasks. Thus, their motivation remained the same throughout the course.

Finally, one more interesting feature is that approximately 10 percent of the students gained full marks in the exercises, although they knew that this did not bring them any extra benefit. They were apparently motivated by the assignments. In addition, the learning environment provided tools for enhancing their performance by resubmitting exercises until they were satisfied with their performance.

\section{OBSERVATIONS ON LATE NIGHT SUB- MISSIONS}

Automatic assessment systems allow students to submit the answers at any time of day. Our systems record the submission times, which shows the following interesting statistics. In Table 1 we see the total number of submissions and the percentage of the passed submissions at different timescales during the Scheme-course in years 1999 and 2000. The data has been collected from the whole semester. As might be anticipated, the number of night submissions is much lower than during the day. However, the most interesting factor is the low number of passed late night submissions, particularly between at 4 and 5 am. Statistically those results are significantly lower than results at other times within the $95 \%$ confidence interval.

\begin{tabular}{|c|r|r|r|r|}
\hline time & $N_{1999}$ & $P_{1999}$ & $N_{2000}$ & $P_{2000}$ \\
\hline \hline $0-1$ & 796 & 60.9 & 697 & 50.9 \\
$2-3$ & 304 & 58.9 & 353 & 60.9 \\
$4-5$ & 135 & 38.5 & 83 & 39.8 \\
$6-7$ & 124 & 46.8 & 137 & 58.4 \\
$8-9$ & 644 & 62.6 & 503 & 54.5 \\
$10-11$ & 2445 & 63.6 & 2085 & 55.6 \\
$12-13$ & 2066 & 63.0 & 2128 & 54.9 \\
$14-15$ & 2406 & 59.8 & 3137 & 57.9 \\
$16-17$ & 2874 & 61.6 & 3002 & 59.5 \\
$18-19$ & 1893 & 60.2 & 1650 & 56.0 \\
$20-21$ & 1586 & 58.8 & 1170 & 52.5 \\
$22-23$ & 1420 & 59.2 & 1116 & 55.3 \\
\hline
\end{tabular}

Table 1: Number of submitted assignments and percentage of passed assignments at different times in 1999 and 2000

A closer investigation of the late night submissions between 3 and 6 am revealed that their distribution was very uneven. There were five deadlines during the semester, every two weeks, each one at noon. Most of these submissions took place in the last night before the deadline. This suggests that we should close the feedback system at night in order to discourage students working with programs in the last night. Clearly, we cannot prevent last minute submissions, but, perhaps, we should discourage working around the clock the previous night.

Another interesting observation shows that the earlier the works were submitted, the better grades were achieved. The best results were achieved by students who submitted their programs more than two weeks before the deadline; the closer the deadline comes, the lower the score received from their first submission.

\section{CONCLUSION}

The recent trend towards teaching large numbers of students has forced us to examine ways automatically to assess students' performance. Our experience is that such systems can be built and adopted for large courses successfully. A number of interesting points arise.

- Resubmission is an important aid for learning. However, the course should be set up to keep the students' motivation for using it high.

- Challenging assignments are important for motivation.

- Grading policy should motivate learners to solve more assignments.

- Automatic assessment provides an easy way to support 24/7 service for students. However, such open learning environments can lead to poor results due to the fact that some students try to complete assignments the night before the deadline. 
In general, automatic assessment is a good tool for promoting learning and easing the teacher's work. However, it can offer much more, since using these techniques allows us to monitor students' learning process to a greater degree than plain results. By investigating the submission times and frequencies, we can obtain new information on their behaviour. Yet more can be achieved when we tune the systems so that we can obtain records and statistics on errors and how they are solved.

\section{REFERENCES}

[1] R. S. Baker, M. Boilen, M. T. Goodrich, R. Tamassia, and B. A. Stibel. Testers and visualizers for teaching data structures. In Proceedings of the 30th SIGCSE Technical Symposium on Computer Science Education, pages 261-265, New Orleans, LA, USA, 1999. ACM.

[2] S. Benford, E. Burke, E. Foxley, N. Gutteridge, and A. M. Zin. Ceilidh: A course administration and marking system. In Proceedings of the International Conference of Computer Based Learning, Vienna, Austria, 1993.

[3] J. English and P. Siviter. Experience with an automatically assessed course. In Proceedings of The 5th Annual SIGCSE/SIGCUE Conference on Innovation and Technology in Computer Science Education, ITiCSE'00, pages 168-171, Helsinki, Finland, 2000. ACM.

[4] J. Hyvönen and L. Malmi. Trakla - a system for teaching algorithms using email and a graphical editor. In Proceedings of HYPERMEDIA in Vaasa, pages 141-147, Vaasa Institute of Technology, Vaasa, Finland, 1993.

[5] D. Jackson. A semi-automated approach to online assessment. In Proceedings of The 5th Annual SIGCSE/SIGCUE Conference on Innovation and Technology in Computer Science Education, ITiCSE'00, pages 164-167, Helsinki, Finland, 2000. ACM.

[6] D. Jackson and M. Usher. Grading student programs using assyst. In Proceedings of 28th ACM SIGCSE Tech. Symposium on Computer Science Education, pages 335-339, San Jose, California, USA, 1997. ACM.
[7] A. Korhonen and L. Malmi. Algorithm simulation with automatic assessment. In Proceedings of The 5th Annual SIGCSE/SIGCUE Conference on Innovation and Technology in Computer Science Education, pages 160-163, Helsinki, Finland, 2000. ACM.

[8] A. Korhonen, L. Malmi, P. Myllyselkä, and P. Scheinin. Does it make a difference if students exercise on the web or in the classroom? In Proceedings of The 7th Annual SIGCSE/SIGCUE Conference on Innovation and Technology in Computer Science Education, ITiCSE'02. 2002. ACM.

[9] A. Korhonen, L. Malmi, and R. Saikkonen. Matrix concept animation and algorithm simulation system. In Proceedings of The 6th Annual SIGCSE/SIGCUE Conference on Innovation and Technology in Computer Science Education, ITiCSE'01, page 180, Canterbury, United Kingdom, 2001. ACM.

[10] A. Korhonen, J. Nikander, R. Saikkonen, and P. Tenhunen. Matrix - algorithm simulation and animation tool. http://www.cs.hut.fi/Research/Matrix/, November 2001.

[11] D. C. Phillips. Constructivism in Education. Opinions and Second Opinions on Controversial Issues. Ninety-ninth Yearbook of the National Society for the Study of Education. Part 1. The University of Chicago Press, Chicago, Illinois, USA, 2000.

[12] K. A. Reek. The try system or how to avoid testing student programs. In Proceedings of SIGCSE'1989, pages 112-116. 1989. ACM.

[13] R. Saikkonen, L. Malmi, and A. Korhonen. Fully automatic assessment of programming exercises. In Proceedings of The 6th Annual SIGCSE/SIGCUE Conference on Innovation and Technology in Computer Science Education, ITiCSE'01, pages 133-136, Canterbury, United Kingdom, 2001. ACM. 